\title{
Adaptation and learning of molecular networks as a description of cancer development at the systems-level: potential use in anti-cancer therapies
}

\author{
Dávid M. Gyurkó ${ }^{1}$, Dániel V. Veres ${ }^{1}$, Dezső Módos ${ }^{1,2,3}$, Katalin Lenti ${ }^{2}$, Tamás Korcsmáros ${ }^{3}$ \\ and Peter Csermely ${ }^{1,1}$
}

${ }^{1}$ Semmelweis University, Department of Medical Chemistry, Tuzolto u. 37-47, H-1094 Budapest, Hungary; ${ }^{2}$ Semmelweis University, Department of Morphology and Physiology, Faculty of Health Sciences, Vas u. 17, H1088, Budapest, Hungary; ${ }^{3}$ Eötvös Loránd University, Department of Genetics, Pazmany P. s. 1c, H-1117

Budapest, Hungary

\begin{abstract}
There is a widening recognition that cancer cells are products of complex developmental processes. Carcinogenesis and metastasis formation are increasingly described as systems-level, network phenomena. Here we propose that malignant transformation is a two-phase process, where an initial increase of system plasticity is followed by a decrease of plasticity at late stages of carcinogenesis as a model of cellular learning. We describe the hallmarks of increased system plasticity of early, tumor initiating cells, such as increased noise, entropy, conformational and phenotypic plasticity, physical deformability, cell heterogeneity and network rearrangements. Finally, we argue that the large structural changes of molecular networks during cancer development necessitate a rather different targeting strategy in early and late phase of carcinogenesis. Plastic networks of early phase cancer development need a central hit, while rigid networks of late stage primary tumors or established metastases should be attacked by the network influence strategy, such as by edgetic, multi-target, or allo-network drugs. Cancer stem cells need special diagnosis and targeting, since their dormant and rapidly proliferating forms may have more rigid, or more plastic networks, respectively. The extremely high ability to change their rigidity/plasticity may be a key differentiating hallmark of cancer stem cells. The application of early stage-optimized anti-cancer drugs to late-stage patients may be a reason of many failures in anti-cancer therapies. Our hypotheses presented here underlie the need for patient-specific multi-target therapies applying the correct ratio of central hits and network influences - in an optimized sequence.
\end{abstract}

Key words: adaptation; anti-cancer therapies; cancer attractors; cancer development; epithelial-mesenchymal transition; interactome; networks; signaling Abbreviations: BRAF, B-Raf protein; BRD4, bromodomain-containing protein 4; CDK6, cyclin-dependent kinase 6; ERBB1, epidermal growth factor receptor; ERG, ETS-family oncogenic transcription factor; ERK, extracellular signal regulated protein kinase; FOS, FBJ murine osteosarcoma viral oncogene homolog; FOXO3A, forkhead family transcription factor; IRS1, insulin receptor substrate 1; MMP2, matrix metalloproteinase 2; mTORC1, mammalian target of rapamycin complex 1; MYC, myelocytomatosis viral oncogene homolog protein; NES, nestin intermediate filament protein; p53, TP53 tumor suppressor protein; PDGFR, platelet-derived growth factor receptor; PI3K, phosphatidyl-inositol-3'-kinase; PKM2, pyruvate kinase M2 isoform; RAS, small GTPase protein; RHOA, RAShomolog gene family member A; TGFBR, Transforming growth factor- $\beta$ receptor; TNC, tenascin $\mathrm{C}$ protein.

In this paper first we will describe cancer development as a two-phase phenomenon characterized by a first increased than decreased plasticity (or in alternative wording: by a first decreasing than increasing rigidity) at the systems-level. We will propose that cancer stem cells have the unique property to induce rapid and gross changes of their network plasticity/rigidity. Next, we will list the cancerspecific properties of molecular networks highlighting the adaptation of network structure and dynamics in various stages of cancer development as a model of cellular learning. Finally, we will highlight that different network-related anti-cancer strategies are needed to target early and late stage cancer cells, as well as cancer stem cells, and will list the possibilities to target all these cell communities.

\section{Development of cancer as a learning process of increasing and then decreasing plasticity at the systems-level}

Cancer cells are products of a complex cell transformation process. The starting steps of this process are often mutations or DNA-rearrangements, which destabilize the former cellular phenotype. As a result, a cell population with a large variability in

${ }^{1}$ Corresponding author; Csermely.Peter@med.semmelweis-univ.hu 
chromatin organization (including DNA-hypomethylation, histone modification and chromatin structure), gene expression patterns and interactome composition is formed. This heterogeneous cell population is characterized by an increased level of stochastic processes (noise), phenotypic plasticity [1-6], and by an increase in the network entropy of protein-protein interaction networks (the definitions of local and more global variants of network entropy see in [7-9]). Higher degree-entropy of signaling networks was found to correlate with lower survival of prostate cancer patients [9], which underlies both the medical relevance and the generality of these changes. Increased heterogeneity may help survival not only at the level of individual cells, but also by allowing the formation of cooperating inter-cellular networks of cancer cells $[10,11]$. This cooperative, paracrine cross-regulation may change to a selfish, autocrine development later $[12,13]$.

In many general aspects, the development of cancer cells resembles to that resulting in changes of cellular phenotypes e.g. during embryogenesis. Major steps of the epithelial-mesenchymal transition (where epithelial cells lose both their apical/basal polarity and original cellular contacts, gain the ability to transverse the extracellular matrix, and ultimately contribute to tissues other than the original epithelial sheet) correspond well to the major stages of metastasis formation [14,15].

Here we argue that malignant transformation is essentially a two-phase process. We propose that the first phase of malignant transformation is characterized by an increase of plasticity in the protein-protein interaction, signaling and other networks of transformed cells, which is followed by decrease of network plasticity (Fig. 1), where network plasticity (or in other words network flexibility) is related to the degrees of freedom of network nodes [16]. The initial stage may correspond to "clonal expansion", and the appearance of tumor initiating cells. The late stage may represent either late-stage primary tumor cells, or metastatic cells, which already settled in their novel tissue environment.

Cancer stem cells [17] can possess both types of the above duality. Cancer stem cells can display a dormant phenotype, which is not rapidly proliferating, and has a more rigid structure of its protein-protein interaction, signaling and other networks than that of rapidly proliferating early stage tumor cells. Importantly, cancer stem cells may also possess a rapidly proliferating phenotype [17], which may have a highly plastic network structure. Therefore, cancer stem cells are not discriminated by the plasticity of their actual network structures (called as structural plasticity [16]), but by their especially high ability to modulate the plasticity of their networks (called as dynamic plasticity [16]) according to the needs of the environment. It is important to note that the specially high ability of cancer stem cells to modulate the plasticity of their networks is in accordance with the original cancer stem cell definition of tumor initiation after serial dilutions [17]. A highly increased ability of plasticity modulation (which results in an increased level of evolvability) may prove to be a major discriminatory hallmark of cancer stem cells. Importantly, this increased plasticity modulation ability may be a key reason why anti-cancer therapies often induce cancer stem cells instead of killing or transforming them.

There are many signs of the initial increase of system plasticity during cancer development, such as the increased heterogeneity, noise and entropy mentioned before. Tumor initiating cells showed a larger plasticity also at the level of physical deformability $[18,19]$. However, at late stage carcinogenesis of the primary tumor, or when tumor cells already established metastases and were incorporated to a more stable tissue environment, the systems-level plasticity of cancer cells may decrease again.

The cancer-specific, metastable states, which were termed as "cancer attractors" by Stuart Kauffman in $1971[3,20]$ may be especially typical to these later stages of cancer development. Additionally, early stages of cancer development may be characterized by numerous "shallow" cancer attractors developing a more plastic structural network of the cell residing in transit on this relatively smooth state space environment, while late stages of tumor development may involve fewer but "deeper" cancer attractors, where the cancer cell becomes stabilized in this more rough state space environment. The dual change described above corresponds well to various steps in the transition to cancer attractors, since cancer cells should first cross a barrier in the (epigenetic) fitness landscape, which might be lowered by mutations or epigenetic changes [3], but still requires a transient destabilization of the transforming cell, which leads to a more plastic phenotype with all the phenotypic characteristics described before. The phenotype of the already established, late-stage cancer cells is still more plastic and immature than that of normal cells, but may often be more rigid than the phenotype of the cells in the intermediate stages of carcinogenesis (Fig. 1).

Such a biphasic change resembles to that of cell differentiation processes, where an initial increase of entropy of chromosomal order and co-regulated gene expression pattern is followed by a later decrease [21]. An analogous set of events happens in cellular reprogramming, where single-cell studies revealed that an early stage, very heterogeneous, stochastic phase is followed by a late phase, which is programmed by a hierarchical set of transcription factors [22]. The recently discovered super-enhancers [23,24] may characterize this second, consolidated phase of phenotype rearrangement. Importantly, a more ordered system is generally less controllable than a disordered one [25], which warns that (A) therapeutic interventions of early stage of carcinogenesis are more efficient than those against late-stage tumors; (B) late-stage tumors should be attacked by a fully different strategy than early-stage tumors preferring indirect targets having a smaller centrality in molecular networks, which cause less side effects and toxicity in these more rigid, late-stage cancer-specific networks/cellular systems, see Fig. 1 and [26].

\section{Network segments participating in adaptive processes}

The cancer development process is increasingly described as a systems-level, network phenomenon [27,28]. Applying this description to the cancer development stages described above, in this section we summarize the adaptation options of network structures in general. In Section 3, we will describe the cancer-specific network adaptation events.

Adaptation of networks is often described as "network evolution", where the term refers to changes in the network contact structure (e.g. changes of parameters like network connectivity, edge weights, diameter, centrality, motifs or modules $[29,30])$. The identification of these changes has a predictive potential both in retrospect and about future development of the complex system represented by the network. Network evolution may follow different timescales varying from microseconds to decades or more [31-33]. Thus the assessment of molecular network changes in the progression of cancer requires a very careful selection of timeframes both in clinical sampling and in network analysis.

Network dynamics extends the frame of network evolution to the refined changes of network nodes (e.g. individual proteins) when transmitting signals, or participating in their cellular function requiring a concerted action of multiple proteins. Network dynamics is strongly related to the underlying network topology. Transitions to a new state, as it was seen in tumor development [34], have key importance in network dynamics.

Hubs (i.e. nodes with much more neighbors than the average) are key determinants of local network topology [35]. A key example of these highly connected hubs is the p53 protein. The p53 tumor suppressor is indeed a key regulator of cancer-related molecular 


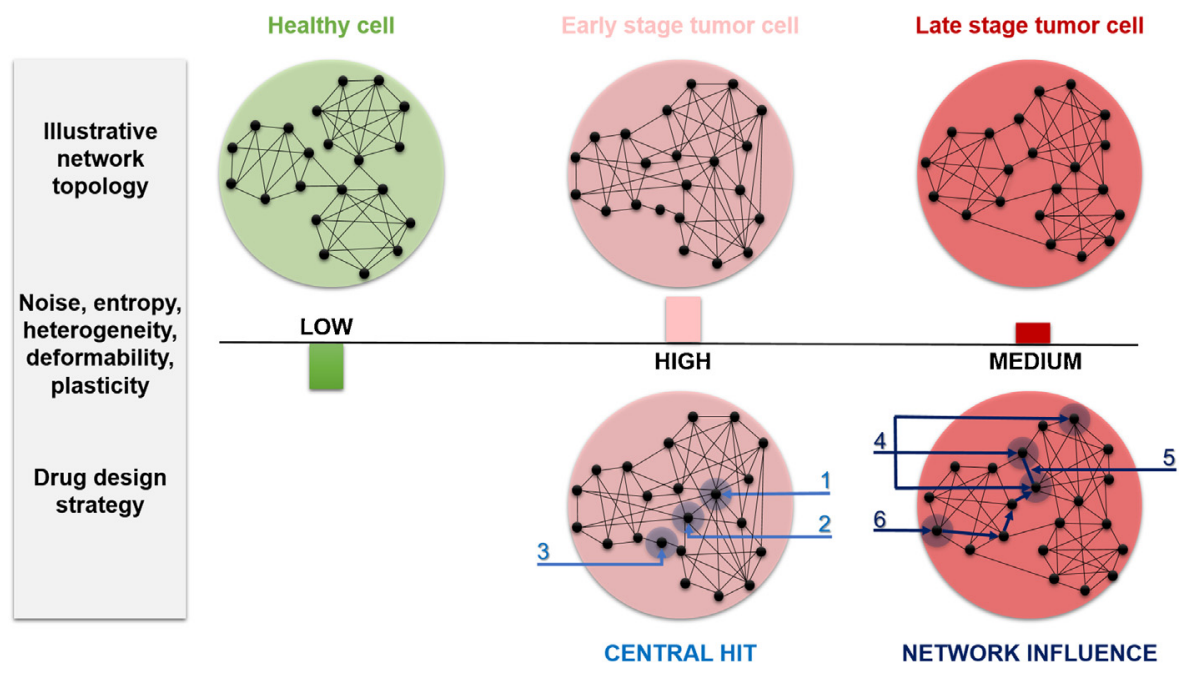

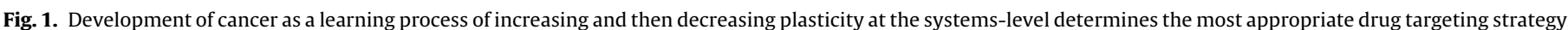

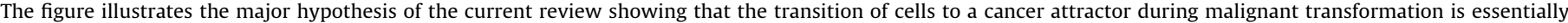

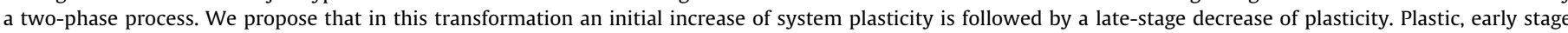

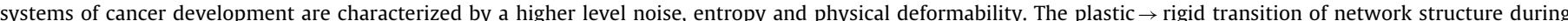

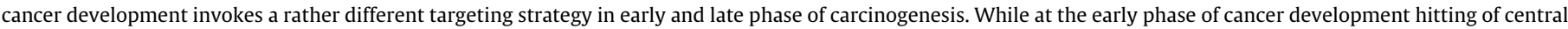

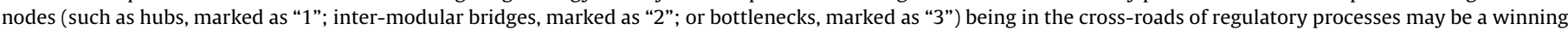

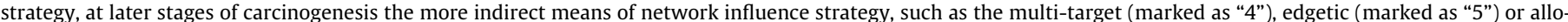

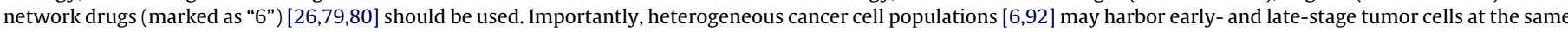

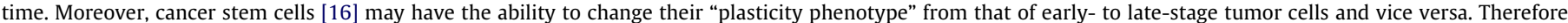

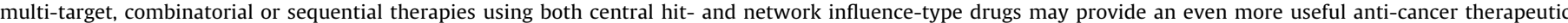
modality than previously thought

networks [36-38]. Many different pathways may converge on hubs, which are often evolutionary conserved proteins [39]. Reversely, a hub often acts as a distributor of perturbations. Network motifs are repeated local patterns of network topology typically consisting of 3 to 6 nodes. Negative feedback loops and feed-forward loops are significant motifs in adaptation $[40,41]$.

Network modules are groups of nodes connected more densely to each other than to the rest of the network. In molecular networks a spatial or temporal module often means a functional unit [42]. Altered modularity was shown as a predictive measure in breast cancer prognosis [43]. Most modules of cellular networks have a high overlap with each other [44,45]. Modules of the yeast protein-protein interaction network are becoming more condensed and displaying smaller overlaps upon stress [46]. In extreme cases stress may result in the disintegration of the network leading to the death of the organism, which may be an important part of the network-level mechanism of action of several anti-cancer drugs.

Modules may segregate network segments, which are more plastic and/or more rigid. While rigid network segments preserve the result of a past adaptive process often displaying an optimized function, flexible network segments are capable of plastic adaptation to present or future challenges of the environment. While rigid network segments are able for fast signal transmission without a large dissipation, plastic network segments have a slower signal transmission and a larger dissipation [16,26,47].

Bridges are node-pairs connecting modules, and a bottleneck is a key inter-modular node. Network perturbations have to propagate through these nodes, therefore bridges and bottlenecks are important points of regulation and adaptation $[48,49]$. The socalled creative nodes [50] are joining multiple modules in a highly dynamic fashion. Creative nodes connect functionally distinct modules. Therefore, the abundance of these exceptionally unpredictable nodes may be a key regulator of "adaptation-speed" and related system plasticity and evolvability. Fig. 2 illustrates the most important structural elements of network adaptation.

\section{Cancer-specific properties of molecular networks}

After the description of key network segments participating in adaptive processes in general, here we summarize the current knowledge on the most important changes of molecular networks in cancer development. Table 1 highlights a few proteins playing a key role in cancer-related molecular networks.

Protein structure networks have nodes of proteins as amino acids, where the edge weight depends on the distance of two neighboring amino acids in the 3D structure of the protein. Detailed studies on the network representation of cancer-specific proteins and their mutations are missing. However, recurrent findings showed that intrinsically disordered proteins play an important role in cancer-specific cellular events [51-53]. Additionally, cancerrelated proteins have smaller, more planar, more charged and less hydrophobic binding interfaces than other proteins, which may indicate a low affinity and high specificity of cancer-related interactions [54]. An increased "conformational noise" in cancer represented by intrinsically disordered proteins and by low affinity interactions would be in agreement with the more plastic network structure of tumor initiating cells. Our hypothesis would predict a decreased expression of disordered proteins or proteins with low affinity interactions in the late-phase of cancer development (i.e. in late stage primary tumors, or when metastatic cells already settled in the novel tissue environment) as opposed to the early phases of "clonal expansion", or cancer stem cell formation, where the expression of these proteins may be higher.

Protein-protein interaction networks (interactomes) have the individual proteins (or their domains) as nodes, and their physical interactions as edges. Cancer-specific interactomes may be constructed by taking into account protein abundances, or (as a first approximation) incorporating mRNA expression patterns $[48,55,56]$. A microarray gene profiling study showed that genes with elevated expression are coding well-connected proteins, while suppressed genes code less connected proteins in lung 


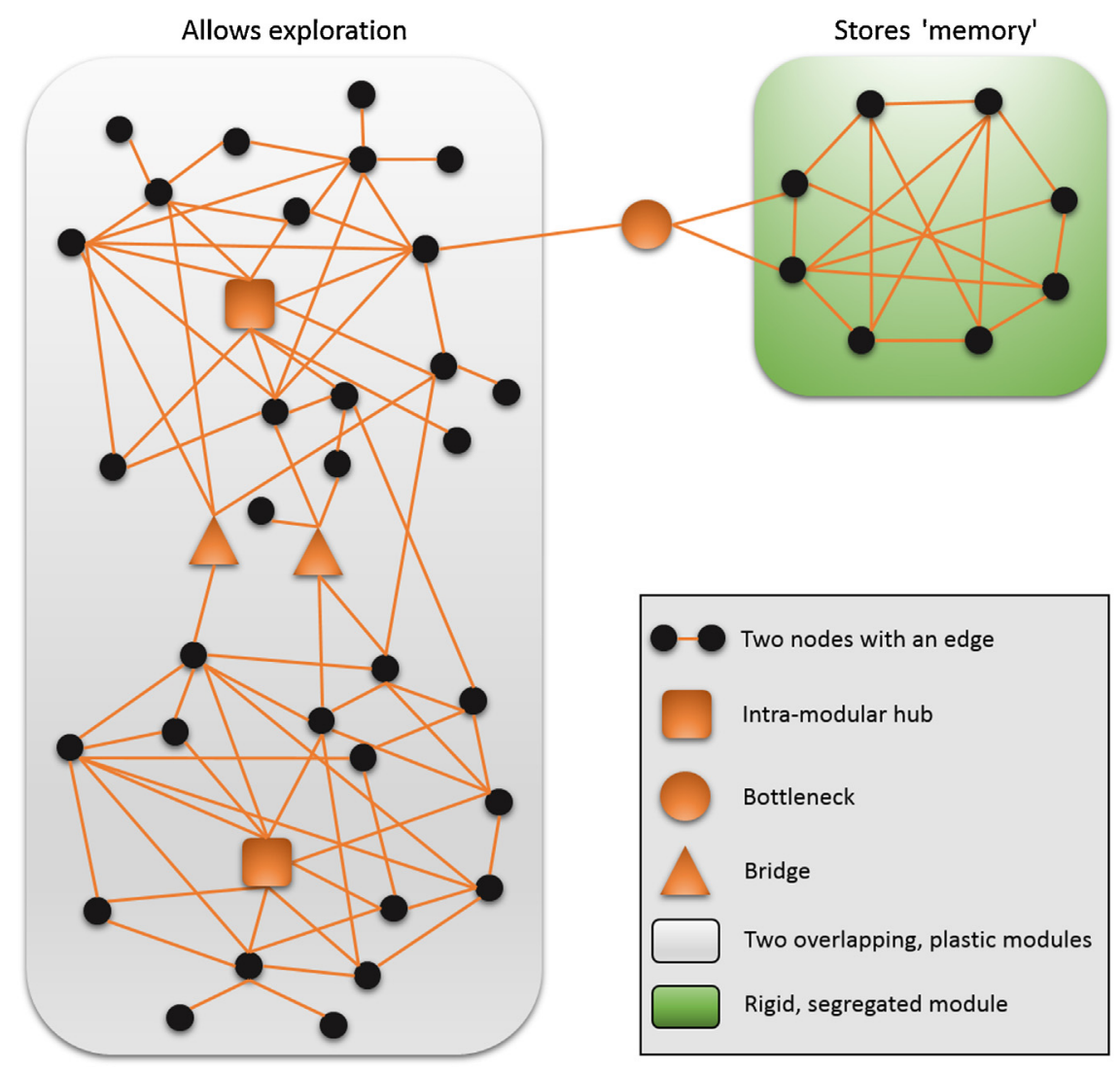

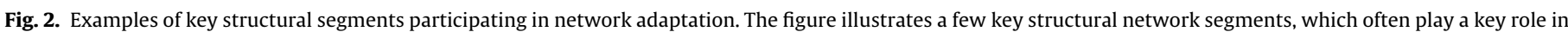

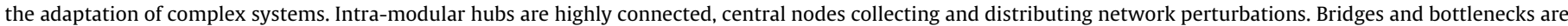

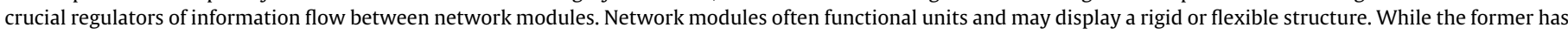

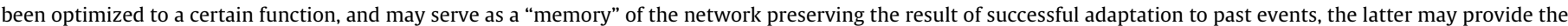
plasticity promoting adaptive processes in the present or future.

squamous cancer [57]. Interactome-based studies indicated that proteins with cancer-specific mutations are hubs with a tendency to form a "rich-club", i.e. being connected with each other [58], and are located in the center of modules or create bridges between them [59]. Inter-modular hubs, such as IRS1 or BRAF, have a particularly important role in oncogenesis [43]. Cancer-related proteins tend to form transient interactions and usually involved in multiple pathways [54]. Dynamical rearrangements of interactome segments may contribute to the changes in plasticity during cancer development. Gene-expression enriched protein-protein interaction networks of 6 cancer types showed an increased network entropy, if compared to interactomes of healthy cells [8]. Increased network-entropy may be an important ingredient of the increased plasticity of malignant cells, and may significantly contribute to the increased robustness against stress and other environmental stimuli.
The autonomy of the cancer cell is based mainly on the changes in its signaling network, formed by interconnected signaling pathways including its gene regulatory network [60]. Changes in the expression level of signaling proteins in cancer cells may cause the activation of major cancer-related pathways, and can rewire the whole signaling network, as it was shown in the case of the epidermal growth factor receptor (ERBB1) signaling network [61]. Cross-talks between the individual signaling pathways are able to create a high number of new input-output combinations increasing the plasticity of the signaling network [2]. Earlier, we found a significant change in the expression level of specific cross-talking proteins in hepatocellular carcinoma leading to a highly different, malignant network of signaling pathways [62]. The up-regulation and cross-talks of RAS-ERK and PI3K-mTORC1 pathways play a key role in the progression of several tumor types [63]. The regulation of signaling cross-talks highly depends on the spatial organization of

Table 1

Network drug target options.

\begin{tabular}{|c|c|c|c|}
\hline Molecular network type & Possible drug targets & Cancer-specific network properties of possible drug targets & References \\
\hline Protein-protein interaction network & p53, CDK6 & Hub inactivation, bottleneck dysfunction & {$[36,49]$} \\
\hline \multirow[t]{2}{*}{ Signaling network } & ERBB1 & Edgetic perturbation of receptor tyrosine kinase pathway & [61] \\
\hline & PDGFR, TGFBR & $\begin{array}{l}\text { Cross-talks of different pathways during } \\
\text { epithelial-mesenchymal transition }\end{array}$ & [95] \\
\hline Genetic interaction network & MYC, NES, MMP2, FOS, TNC & Hub over-activation, hub-driving node under-activation & [96] \\
\hline Chromatin network & BRD4 & Super-enhancer participating hub & {$[23]$} \\
\hline \multirow[t]{2}{*}{ Metabolic network } & Fumarate hydratase, Succinate & Targeting the Warburg effect, & [97] \\
\hline & $\begin{array}{l}\text { dehydrogenase, } \\
\text { PKM2 }\end{array}$ & Increased metabolic flux & [98] \\
\hline
\end{tabular}


the cell involving differences in molecular crowding [64], scaffold proteins [65], protein translocation [66] or membrane association [67]. Increased network entropy was found in the signaling network of the metastasizing phenotype of breast cancer, if compared to non-metastasizing cells [7]. This may indicate the increased plasticity of cancer-specific signaling in the early phases of tumor development. Our recent studies showed that increased plasticity of signaling networks characterizes the initial stages of tumor development (such as adenomas) better than later stages of malignant transformation (such as carcinomas; Dezső Módos, Katalin Lenti, Tamás Korcsmáros and Péter Csermely, in preparation).

Transcriptional regulation is often executed by a hierarchical cascade of transcriptional factors. The recently discovered superenhancers $[23,24]$ showed that master transcription factors may often establish a cancer-specific super-enhancer locus. Whether the organization of these super-enhancer regions represents a latephase of carcinogenesis, and is preceded by a very heterogeneous, stochastic early phase, like that in cell reprogramming [22], is an open question of future studies.

Chromatin networks are formed by connections between distant DNA-segments, and can be used for the explanation of the chromosomal alterations in cancer [68]. The overexpression of the prostate cancer oncogenic transcription factor, ERG induced a profound effect on chromatin network configuration [69]. Further studies are needed to assess the cancer-specific alterations of transcription-related chromatin networks, such as that describing the connection pattern established by the active RNA-polymerase II-complex [70].

Regulation or mutations of microRNAs play a crucial role in the regulation of cancer-specific gene expression [71]. MicroRNAs can function either as tumor suppressors, such as the let-7 family members targeting the RAS signaling pathway in breast cancer [72], or as oncogenes, like the miR-155 targeting key breast cancer genes, such as FOXO3A or RHOA [73]. The analyses of cancer-specific microRNA networks showed more disjointed subnetworks than in normal tissues [74]. Disjoint modules decrease the interdependence of modular changes, and also serve as an adaptive response in stress [46]. Disjoint modules confer a greater plasticity to the whole system.

Metabolic networks consist of major metabolites as nodes connected by specific enzymatic reactions converting them to each other [75]. Global changes in metabolic networks contribute to the Warburg-effect, and are driven by key enzymes such as the PKM2 isoenzyme of pyruvate kinase [76,77].

The stage-specific changes of molecular networks in tumorigenesis are still largely unexplored, because we usually have detailed systems-level network data only on the two endpoints, i.e. the healthy tissue and the developed late tumors. Therefore, the assessment of consecutive changes in network structure and dynamics during intermediate, consecutive steps of malignant cell and metastasis formation are key steps of future systems-level analysis of cancer development. Network analysis of cancer stem cells will provide a special understanding of both plasticity modulation and evolvability at the systems-level.

\section{Network-related anti-cancer therapies}

It is a growing challenge to identify new drug targets and efficient combination of drugs in order to design new anti-cancer therapies with less toxicity, side-effects and resistance development. As we have shown in the preceding section molecular networks at various levels greatly improved our systems-level understanding of tumor initiation and progression $[26-28,78]$.

Recently a dual network strategy, the central hit strategy and the network influence strategy, was described to target various diseases [26]. In cancer both strategies may be used. Using the central hit strategy our aim is to damage the network integrity of the malignant cell in a selective manner. This may be the most important strategy to attack tumor initiating cells at their first stage of development (e.g. early stages of malignant transformation of metastasis formation). Using the network influence strategy we would like to shift back the malfunctioning network to its normal state. This may be the most important way to shift back tumor cells from their cancer attractors reached in late stages of their development (e.g. late stage primary tumors or after the metastatic cells settled to their new tissue environment). The central hit strategy is applied against a population of highly destabilized cells having a very plastic network structure. Efficient targeting of these systems requires the targeting of their central nodes/edges. In contrast, the network influence strategy is applied to cells, which have a much more rigid network structure than the networks of highly undifferentiated or dedifferentiated cells. Targeting the central nodes/edges of systems having a low plasticity may easily 'over-saturate' the system causing side-effects and toxicity. Therefore, the network influence strategy often needs an indirect approach, where e.g. neighbors of the real target are targeted (allo-network drugs [79]). In another indirect approach of the network influence strategy multiple targets are targeted at the same time 'mildly' (using multitarget drugs [80]), and their indirect and/or superposing effects lead to the reconfiguration of diseased network state back to normal.

The central hit strategy often uses the key central position of inter-modular hubs. These central nodes are often important oncogenes serving as targets in drug development [43]. Similarly, in signaling networks protein or microRNA hubs $[48,57,58,81,82]$, inter-modular positions of cross-talks [83], bottlenecks [84] as well as multi-pathway proteins offer important target candidates $[43,62]$ as central hits.

However, the central hit strategy may often attack nodes, which are so central that their inhibition damages key functions of healthy cells. As an example, mTOR, the mammalian target of rapamycin is an important multi-pathway protein, which mutates in most of the tumors and thus causes hyper-active phenotype [85]. Because of the super-central position of mTOR, edgetic drugs targeting single mTOR interactions have a much more selective effect [86] than targeting all mTOR interactions by conventional drugs [87]. Another example of edgetic anti-cancer therapies is the inhibition of p53/MDM2 connection by nutlins, which liberate the tumorsuppressor effect of $\mathrm{p} 53$ from MDM2-induced inhibition [88].

The network influence strategy often implies multi-target attacks [80]. These attacks may allow the simultaneous targeting of two or more peripheral nodes instead of targeting a single, central node [25]. Such combinatorial or multi-target therapies can be identified using interactomes, signaling or metabolic networks $[89,90]$ and led to the design a simultaneous targeting therapy e.g. in colorectal cancer [91]. The systematic development of allonetwork drugs [79] acting at the neighborhood of real targets and specifically transmitting their signals to them requires a more detailed knowledge of both indirect targeting and the molecular details of allosteric action, and therefore remains and major challenge of future studies.

\section{Network adaptation in various stages of cancer development as a key aspect of anti-cancer therapies}

Here we propose that the large structural changes of molecular networks during cancer development require a rather different targeting strategy in early and late phase of carcinogenesis (Fig. 1). While at the early phase of cancer development hitting of central nodes in various molecular networks (such as inter-modular hubs being in the cross-roads of regulatory processes) may become a 
winning strategy, the very same pharmacological intervention may become useless at a later stage of carcinogenesis, where the primary tumor was already established, or the metastasized cells have been settled in their new tissue environment. These late-stage tumor cells should be attacked by the more indirect means of network influence strategy, such as by edgetic, multi-target, or allo-network drugs $[26,79,80]$.

The analysis of Rajapakse et al. [25] offers important clues on targeting molecular networks during cancer development. Referring to the general observation that symmetrical networks are more uncontrollable, they hypothesize that the highly heterogeneous, highly plastic, intermediate state of tumor-initiating cells right before or at attaining their metastatic potential is the most difficult to attack by well-targeted external inputs. This is the phase, where only the central-hit strategy may be applied. However, at late stage primary or metastatic tumors, where the underlying network structures became more asymmetric again (Dezső Módos, Katalin Lenti, Tamás Korcsmáros and Péter Csermely, in preparation) only the indirect effects of the network influence strategy may be successful.

Very importantly, cancers often harbor cancer stem cells, which may be induced by conventional anti-cancer therapies, themselves [16]. These stem cell-like tumor cell subpopulations possess an extraordinarily high ability to change their plasticity. When cancer stem cells acquire a more dormant state, they may change their networks from a more plastic to a more rigid structure. On the contrary, cancer stem cells may have a transition from a more rigid to a more plastic network structure, if they form early tumor progenitor cells. This large level of dynamic plasticity may be a major reason of the development of drug resistance in many cancer cases.

The presence of highly heterogeneous cell populations (including various forms of cancer stem cells) in a cancer patient, as well as the extreme heterogeneity of patient subtypes [6,92] all give a particular importance of sequential multi-target therapies, where multiple drug treatments are given in a particular order using a well-designed temporal pattern of consecutive treatments. In agreement with Kitano [1] and many key results in the field (e.g. when epidermal growth factor receptor inhibitors sensitized cancer cells to subsequent DNA-damage by unmasking an apoptotic pathway in breast cancer see the work of [93] and the review of Huang and Kauffman in this issue) we strongly believe that sequential multi-target therapy will be the major mode of intervention in cancer therapies of the future.

\section{Conclusions and perspectives}

In conclusion, in this review we proposed that malignant transformation is a two-phase process, where an initial increase of system plasticity is followed by a decrease of plasticity at late stages of carcinogenesis of primary tumor cells or of metastatic cells already settled in their novel tissue environment. The increased systems-level plasticity of cancer initiating cells is characterized by an increased

- level of stochastic processes (noise);

- network entropy;

- "conformational noise" represented by intrinsically disordered proteins and by low affinity interactions;

- phenotypic plasticity;

- cell heterogeneity;

- physical deformability and

- dissociation of network modules (Fig. 1).

Our hypothesis would predict a decrease in all the above parameters in the late-phases of cancer development as opposed to the early phases described above. In late phase carcinogenesis the increase of system rigidity may be accompanied by an increased hierarchy of regulatory processes.

The large structural changes of molecular networks during cancer development require a rather different targeting strategy in early and late phase of carcinogenesis. Importantly, a more ordered system is generally less controllable than a disordered one [25], which warns that (A) therapeutic interventions of the early, more plastic stage of carcinogenesis are more efficient than those against late-stage tumors; (B) late-stage tumors should be attacked by an entirely different strategy than early-stage tumors, see Fig. 1 and [26]. Besides other therapeutic modalities (such as surgery, radiotherapy etc.) at the early phase of cancer development hitting of central nodes of cancerous molecular networks (such as intermodular hubs being in the cross-roads of regulatory processes) may become a winning strategy killing the very heterogeneous cancer cell population. However, central hit-type pharmacological interventions may become useless at a later stage of carcinogenesis. Late stage primary tumors or the already metastasized cells in their new tissue environment should be attacked by the more indirect means of network influence strategy, such as by edgetic, multi-target, or allo-network drugs see Fig. 1 and $[26,79,80]$, since here not the eradication of a large heterogeneity of tumor cells, but the shift of the malignant cellular network to a less malignant state is the desired action.

Regretfully, many in vitro test systems of anti-cancer drug candidates resemble to the plastic cellular systems of early stage cancer development, while disease is usually detected in patients, when it reached the late phase of development [94]. The application of early stage-optimized anti-cancer drugs to late-stage patients may be a reason of many failures in anti-cancer therapies. This situation underlies the importance of systems- level studies of cancer cell development and the network analysis of the data obtained.

Importantly, the initial increase and later decrease of system plasticity during cancer development may appear both at the level of the network of individual cells and at the level of the network of cell populations. Heterogeneous cancer cell populations may harbor early- and late-stage tumor cells at the same time. Moreover, cancer stem cells may have the ability to change their "plasticity phenotype" from that of early- to late-stage tumor cells and vice versa. Therefore, multi-target, combinatorial or sequential therapies using both central hit- and network influence-type drugs may provide an even more useful anti-cancer therapeutic modality than previously thought.

\section{Conflict of interest}

Authors declare no conflict of interest.

\section{Acknowledgements}

Work in the authors' laboratory was supported by research grants from the Hungarian National Science Foundation (OTKAK83314), from the EU (TÁMOP-4.2.2/B-10/1-2010-0013) and a János Bolyai Scholarship to TK.

\section{References}

[1] Kitano H. Cancer as a robust system: implications for anticancer therapy. Nature Reviews Cancer 2004;4:227-35.

[2] Kitano H. Biological robustness. Nature Reviews Genetics 2004;5:826-37.

[3] Huang S, Ernberg I, Kauffman S. Cancer attractors: a systems view of tumors from a gene network dynamics and developmental perspective. Seminars in Cell \& Developmental Biology 2009;20:869-76.

[4] Pujadas E, Feinberg AP. Regulated noise in the epigenetic landscape of development and disease. Cell 2012;148:1123-31.

[5] Greaves M, Maley CC. Clonal evolution in cancer. Nature 2012;481:306-13.

[6] Marusyk A, Almendro V, Polyak K. Intra-tumour heterogeneity: a looking glass for cancer? Nature Reviews Cancer 2012;12:323-34. 
[7] Teschendorff AE, Severini S. Increased entropy of signal transduction in the cancer metastasis phenotype. BMC Systems Biology 2010;4:104-16.

[8] West J, Bianconi G, Severini S, Teschendorff AE. Differential network entropy reveals cancer system hallmarks. Scientific Reports 2012;2:802-9.

[9] Breitkreutz D, Hlatky L, Rietman E, Tuszynski JA. Molecular signaling network complexity is correlated with cancer patient survivability. Proceedings of the National Academy of Sciences of the United States of America 2012;109:9209-12.

[10] Axelrod R, Axelrod DE, Pienta KJ. Evolution of cooperation among tumor cells. Proceedings of the National Academy of Sciences of the United States of America 2006:103:13474-9.

[11] Bonavia R, Inda MM, Cavenee WK, Furnari FB. Heterogeneity maintenance in glioblastoma: a social network. Cancer Research 2011;71:4055-60.

[12] Creixell P, Schoof EM, Erler JT, Linding R. Navigating cancer network attractors for tumor-specific therapy. Nature Biotechnology 2012;30:842-8.

[13] Wu Y, Garmire LX, Fan R. Inter-cellular signaling network reveals a mechanistic transition in tumor microenvironment. Integrative Biology 2012;4:1478-86.

[14] Thiery JP. Epithelial-mesenchymal transitions in tumour progression. Nature Reviews Cancer 2002;2:442-54.

[15] Kalluri R, Weinberg RA. The basics of epithelial-mesenchymal transition. Journal of Clinical Investigation 2009;119:1420-8.

[16] Tang DG. Understanding cancer stem cell heterogeneity and plasticity. Cell Research 2012;22:457-72.

[17] Gáspár ME, Csermely P. Rigidity and flexibility of biological networks. Briefings in Functional Genomics 2012;11:443-56.

[18] Guck J, Schinkinger S, Lincoln B, Wottawah F, Ebert S, Romeyke M, et al. Optical deformability as an inherent cell marker for testing malignant transformation and metastatic competence. Biophysical Journal 2005;88:3689-98.

[19] Zhang W, Kai K, Choi DS, Iwamoto T, Nguyen YH, Wong H, et al. Microfluidics separation reveals the stem-cell-like deformability of tumor-initiating cells. Proceedings of the National Academy of Sciences of the United States of America 2012;109:18707-12.

[20] Kauffman S. Differentiation of malignant to benign cells. Journal of Theoretical Biology 1971;31:429-51.

[21] Rajapakse I, Perlman MD, Scalzo D, Kooperberg C, Groudine M, Kosak ST. The emergence of lineage-specific chromosomal topologies from coordinate gene regulation. Proceedings of the National Academy of Sciences of the United States of America 2009;106:6679-84.

[22] Buganim Y, Faddah DA, Cheng AW, Itskovich E, Markoulaki S, Ganz K, et al. Single-cell expression analyses during cellular reprogramming reveal an early stochastic and a late hierarchic phase. Cell 2012;150:1209-22.

[23] Lovén J, Hoke HA, Lin CY, Lau A, Orlando DA, Vakoc CR, et al. Selective inhibition of tumor oncogenes by disruption of super-enhancers. Cell 2013;153: 320-34.

[24] Whyte WA, Orlando DA, Hnisz D, Abraham BJ, Lin CY, Kagey MH, et al. Master transcription factors and mediator establish super-enhancers at key cell identity genes. Cell 2013;153:307-19.

[25] Rajapakse I, Groudine M, Mesbahi M. Dynamics and control of state-dependent networks for probing genomic organization. Proceedings of the National Academy of Sciences of the United States of America 2011;108:17257-62.

[26] Csermely P, Korcsmaros T, Kiss HJM, London G, Nussinov R. Structure and dynamics of molecular networks: a novel paradigm of drug discovery. A comprehensive review. Pharmacology \& Therapeutics 2013;138:333-408.

[27] Dulbecco R. Cancer progression: the ultimate challenge. International Journal of Cancer Supplement 1989;4:6-9.

[28] Hornberg JJ, Bruggeman FJ, Westerhoff HV, Lankelma J. Cancer: a systems biology disease. BioSystems 2006;83:81-90.

[29] Dorogovtsev S, Mendes J. Evolution of networks. Advances in Physics 2002;51:1079-187.

[30] Holme P, Saramäki J. Temporal networks. Physics Reports 2012;519:97-125.

[31] Pál C, Papp B, Lercher MJ. An integrated view of protein evolution. Nature Reviews Genetics 2006; 7:337-48.

[32] Pazos F, Valencia A. Protein co-evolution, co-adaptation and interactions. EMBO Journal 2008;27:2648-55.

[33] Gelman H, Platkov M, Gruebele M. Rapid perturbation of free-energy landscapes: from in vitro to in vivo. Chemistry 2012;18:6420-7.

[34] Delsanto PP, Romano A, Scalerandi M, Pescarmona GP. Analysis of a "phase transition" from tumor growth to latency. Physical Review E 2000;62:2547-54.

[35] Barabasi AL, Albert R. Emergence of scaling in random networks. Science 1999;286:509-12.

[36] Vogelstein B, Lane D, Levine AJ. Surfing the p53 network. Nature 2000;408:307-10.

[37] Wade M, Li YC, Wahl GM. MDM2, MDMX and p53 in oncogenesis and cancer therapy. Nature Reviews Cancer 2012;13:83-96.

[38] Schwartz JM, Tian K, Rajendran R, Doddananjaiah M, Krstic-Demonacos M. Dynamics of DNA damage induced by pathways of cancer. PLoS ONE 2013;8, in press.

[39] Fraser HB. Modularity and evolutionary constraint on proteins. Nature Genetics 2005;37:351-2.

[40] Milo R, Shen-Orr S, Itzkovitz S, Kashtan N, Chklovskii D, Alon U. Network motifs: simple building blocks of complex networks. Science 2002;298:824-7.

[41] Ma W, Trusina A, El-Samad H, Lim W, Tang C. Defining network topologies that can achieve biochemical adaptation. Cell 2009;138:760-73.

[42] Spirin V, Mirny L. Protein complexes and functional modules in molecular networks. Proceedings of the National Academy of Sciences of the United States of America 2003;100:12123-8.
[43] Taylor IW, Linding R, Warde-Farley D, Liu Y, Pesquita C, Faria D, et al. Dynamic modularity in protein interaction networks predicts breast cancer outcome. Nature Biotechnology 2009;27:199-204.

[44] Horvath S, Zhang B, Carlson M, Lu KV, Zhu S, Felciano RM, et al. Analysis of oncogenic signaling networks in glioblastoma identifies ASPM as a molecular target. Proceedings of the National Academy of Sciences of the United States of America 2006;103:17402-7.

[45] Furlong LI. Human diseases through the lens of network biology. Trends in Genetics 2012;29:150-9.

[46] Mihalik Á, Csermely P. Heat shock partially dissociates the overlapping modules of the yeast protein-protein interaction network: a systems level model of adaptation. PLoS Computational Biology 2011;7:e1002187.

[47] Gyurkó MD, Soti C, Stetak A, Csermely P. System level mechanisms of adaptation, learning, memory formation and evolvability: the role of chaperone and other networks. Current Protein \& Peptide Science 2013;14, in press.

[48] Jonsson PF, Bates PA. Global topological features of cancer proteins in the human interactome. Bioinformatics 2006;22:2291-7.

[49] Yu H, Kim PM, Sprecher E, Trifonov V, Gerstein M. The importance of bottlenecks in protein networks: correlation with gene essentiality and expression dynamics. PLoS Computational Biology 2007;3:e59.

[50] Csermely P. Creative elements: network-based predictions of active centres in proteins and cellular and social networks. Trends in Biochemical Sciences 2008;33:569-76.

[51] Iakoucheva LM, Brown CJ, Lawson JD, Obradović Z, Dunker AK. Intrinsic disorder in cell-signaling and cancer-associated proteins. Journal of Molecular Biology 2002;323:573-84

[52] Uversky VN, Oldfield CJ, Midic U, Xie H, Xue B, Vucetic S, et al. Unfoldomics of human diseases: linking protein intrinsic disorder with diseases. BMC Genomics 2009;10:S7.

[53] Mahmoudabadi G, Rajagopalan K, Getzenberg RH, Hannenhalli S, Rangarajan G, Kulkarni P. Intrinsically disordered proteins and conformational noise: implications in cancer. Cell Cycle 2013:12:26-31.

[54] Kar G, Gursoy A, Keskin O. Human cancer protein-protein interaction network: a structural perspective. PLoS Computational Biology 2009;5:e1000601.

[55] Coulombe B. Mapping the disease protein interactome: toward a molecular medicine GPS to accelerate drug and biomarker discovery. Journal of Proteome Research 2011;10:120-5.

[56] Sardiu ME, Washburn MP. Building protein-protein interaction networks with proteomics and informatics tools. Journal of Biological Chemistry 2011;286:23645-51.

[57] Wachi S, Yoneda K, Wu R. Interactome-transcriptome analysis reveals the high centrality of genes differentially expressed in lung cancer tissues. Bioinformatics 2005;21:4205-8.

[58] Hase T, Tanaka H, Suzuki Y, Nakagawa S, Kitano H. Structure of protein interaction networks and their implications on drug design. PLoS Computational Biology 2009:5:e1000550.

[59] Xia J, Sun J, Jia P, Zhao Z. Do cancer proteins really interact strongly in the human proteinprotein interaction network? Computational Biology and Chemistry $2011 ; 35: 121-5$

[60] Lin C-C, Chen Y-J, Chen C-Y, Oyang Y-J, Juan H-F, Huang H-C. Crosstalk between transcription factors and microRNAs in human protein interaction network. BMC Systems Biology 2012;6:18-30.

[61] Klinke DJ. Signal transduction networks in cancer: quantitative parameters influence network topology. Cancer Research 2010;70:1773-82.

[62] Korcsmáros T, Farkas IJ, Szalay MS, Rovó P, Fazekas D, Spiró Z, et al. Uniformly curated signaling pathways reveal tissue-specific cross-talks, nove pathway components, and drug target candidates. Bioinformatics 2010;26 2042-50.

[63] Mendoza MC, Er EE, Blenis J. The Ras-ERK and PI3K-mTOR pathways: cross-talk and compensation. Trends in Biochemical Sciences 2011:36:320-8.

[64] Lewitzky M, Simister PC, Feller SM. Beyond "furballs" and "dumpling soups" towards a molecular architecture of signaling complexes and networks. FEBS Letters 2012;586:2740-50.

[65] Nussinov R, Ma B, Tsai C-J. A broad view of scaffolding suggests that scaffolding proteins can actively control regulation and signaling of multienzyme complexes through allostery. Biochimica et Biophysica Acta 2013;1834 820-9.

[66] Muscolini M, Montagni E, Palermo V, Di Agostino S, Gu W, Abdelmoula-Souissi $\mathrm{S}$, et al. The cancer-associated K351N mutation affects the ubiquitination and the translocation to mitochondria of p53 protein. Journal of Biological Chemistry 2011;286:39693-702.

[67] Pálfy M, Reményi A, Korcsmáros T. Endosomal crosstalk: meeting points for signaling pathways. Trends in Cell Biology 2012;22:447-56.

[68] Fudenberg G, Getz G, Meyerson M, Mirny LA. High order chromatin architecture shapes the landscape of chromosomal alterations in cancer. Nature Biotechnology 2011;29:1109-13.

[69] Rickman DS, Soong TD, Moss B, Mosquera JM, Dlabal J, Terry S, et al. Oncogenemediated alterations in chromatin conformation. Proceedings of the National Academy of Sciences of the United States of America 2012;109:9083-8.

[70] Sandhu KS, Li G, Poh HM, Quek YL, Sia YY, Peh SQ et al. Large-scale functional organization of long-range chromatin interaction networks. Cell Reports 2012;2:1207-19.

[71] Calin GA, Croce CM. MicroRNA signatures in human cancers. Nature Reviews Cancer 2006;6:857-66.

[72] Yu F, Yao H, Zhu P, Zhang X, Pan Q, Gong C, et al. Let-7 regulates self renewal and tumorigenicity of breast cancer cells. Cell 2007;131:1109-23. 
[73] Mattiske S, Suetani RJ, Neilsen PM, Callen DF. The oncogenic role of miR-155 in breast cancer. Cancer Epidemiology, Biomarkers \& Prevention 2012;21:1236-43.

[74] Volinia S, Galasso M, Costinean S, Tagliavini L, Gamberoni G, Drusco A, et al. Reprogramming of miRNA networks in cancer and leukemia. Genome Research 2010;20:589-99.

[75] Ma H, Goryanin I. Human metabolic network reconstruction and its impact on drug discovery and development. Drug Discovery Today 2008;13: $402-8$.

[76] Shlomi T, Benyamini T, Gottlieb E, Sharan R, Ruppin E. Genome-scale metabolic modeling elucidates the role of proliferative adaptation in causing the Warburg effect. PLoS Computational Biology 2011;7:e1002018.

[77] Yang W, Zheng Y, Xia Y, Ji H, Chen X, Guo F, et al. ERK1/2-dependent phosphorylation and nuclear translocation of PKM2 promotes the Warburg effect. Nature Cell Biology 2012;14:1295-304.

[78] Katoh M, Katoh M. Bioinformatics for cancer management in the post-genome era. Technology in Cancer Research and Treatment 2006;2:73-182.

[79] Nussinov R, Tsai CJ, Csermely P. Allo-network drugs: harnessing allostery in cellular networks. Trends in Pharmacological Sciences 2011;32: 686-93.

[80] Csermely P, Agoston V, Pongor S. The efficiency of multi-target drugs: the network approach might help drug design. Trends in Pharmacological Sciences 2005;26:178-82.

[81] Awan A, Bari H, Yan F, Moksong S, Yang S, Chowdhury S, et al. Regulatory network motifs and hotspots of cancer genes in a mammalian cellular signalling network. IET Systems Biology 2007;1:292-7.

[82] Budd WT, Weaver DE, Anderson J, Zehner ZE. microRNA dysregulation in prostate cancer: network analysis reveals preferential regulation of highly connected nodes. Chemistry \& Biodiversity 2012;9:857-67.

[83] Freudlsperger C, Bian Y, Contag Wise S, Burnett J, Coupar J, Yang X, et al. TGF- $\beta$ and NF- $\kappa B$ signal pathway cross-talk is mediated through TAK1 and SMAD7 in a subset of head and neck cancers. Oncogene 2013;32: 1549-59.

[84] Rosado JO, Henriques JP, Bonatto D. A systems pharmacology analysis of major chemotherapy combination regimens used in gastric cancer treatment: predicting potential new protein targets and drugs. Current Cancer Drug Targets 2011;11:849-69.

[85] Zoncu R, Efeyan A, Sabatini DM. mTOR: from growth signal integration to cancer, diabetes and ageing. Nature Reviews Molecular Cell Biology 2011;12:21-35.
[86] Ruffner H, Bauer A, Bouwmeester T. Human protein-protein interaction networks and the value for drug discovery. Drug Discovery Today 2007;12:709-16.

[87] Carracedo A, Ma L, Teruya-Feldstein J, Rojo F, Salmena L, Alimonti A et al. Inhibition of mTORC1 leads to MAPK pathway activation through a PI3K-dependent feedback loop in human cancer. Journal of Clinical Investigation 2008; 118:3065-74.

[88] Vassilev LT, Vu BT, Graves B, Carvajal D, Podlaski F, Filipovic Z, et al. In vivo activation of the p53 pathway by small-molecule antagonists of MDM2. Science 2004;303:844-8.

[89] Murthy D, Attri KS, Gokhale RS. Network, nodes and nexus: systems approach to multitarget therapeutics. Current Opinion in Biotechnology 2013, http://dx.doi.org/10.1016/j.copbio.2013.02.009, in press.

[90] Sakharkar MK, Shashni B, Sharma K, Dhillon SK, Ranjekar PR, Sakharkar KR. Therapeutic implications of targeting energy metabolism in breast cancer. PPAR Research 2013;2013:109285.

[91] Nibbe RK, Koyutürk M, Chance MR. An integrative-omics approach to identify functional sub-networks in human colorectal cancer. PLoS Computational Biology 2010;6:e1000639.

[92] Sadanandam A, Lyssiotis CA, Homicsko K, Collisson EA, Gibb WJ, Wullschleger $\mathrm{S}$, et al. A colorectal cancer classification system that associates cellular phenotype and responses to therapy. Nature Medicine 2013;19:619-25.

[93] Lee MJ, Ye AS, Gardino AK, Heijink AM, Sorger PK, MacBeath G, et al. Sequential application of anticancer drugs enhances cell death by rewiring apoptotic signaling networks. Cell 2012;149:780-94.

[94] Loscalzo J, Barabasi AL. Systems biology and the future of medicine. Wiley Interdisciplinary Reviews: Systems Biology and Medicine 2011;3:619-27.

[95] Huber MA, Kraut N, Beug H. Molecular requirements for epithelialmesenchymal transition during tumor progression. Current Opinion in Cell Biology 2005; 17:548-58.

[96] Bredel M, Bredel C, Juric D, Harsh GR, Vogel H, Recht LD, et al. Functional network analysis reveals extended gliomagenesis pathway maps and three novel MYC-interacting genes in human gliomas. Cancer Research 2005;65:8679-89.

[97] Resendis-Antonio O, Checa A, Encarnación S. Modeling core metabolism in cancer cells: surveying the topology underlying the Warburg effect. PLoS ONE 2010;5:e12383.

[98] Vazquez A, Markert EK, Oltvai ZN. Serine biosynthesis with one carbon catabolism and the glycine cleavage system represents a novel pathway for ATP generation. PLoS ONE 2011;6:e25881. 